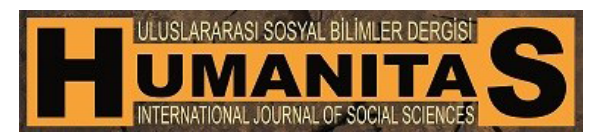

Humanitas,2018; 6(11): 239-255

ISSN:2147-0088X

http://humanitas.nku.edu.tr

DOI: $10.20304 /$ humanitas.391405

\title{
ÜBER DIE HISTORISIERUNG IN ANNA SEGHERS' DAS SIEBTE KREUZ ${ }^{1}$
}

\section{Burcu ÖZTÜRK ${ }^{2}$}

\begin{abstract}
In Anna Seghers' Roman Das siebte Kreuz (1942) wird die Gesellschaft des ,Dritten Reiches' dargestellt. Anna Seghers, deren Bedeutung für die Exilliteratur unbestreitbar ist, kombiniert in ihrem Roman Das siebte Kreuz Fakten und Fiktionen mit Symbolen, um den Nationalsozialismus $\mathrm{zu}$ beschreiben. Im Grunde erzählt Anna Seghers im Siebten Kreuz den kulturellen und historischen Prozess der Menschheit. Auf diese Weise kommt im Text etwas zustande, das als Historisierung bezeichnet werden kann. Im Roman erscheint Geschichte als ein endloser Prozess von Gewalt, in dem es immer Sieger und Besiegte gibt, oder anders ausgedrückt: in dem Sieger immer irgendwann $\mathrm{zu}$ Besiegten werden. Durch die Variante der Historisierung aber gewinnt das Problem, das Seghers darstellt, eine universale Dimension; durch die Zahlensymbolik und die Symbolik des Kreuzes wird dies nurmehr unterstrichen. Deshalb kommt dem Phänomen Historisierung eine so wichtige Bedeutung zu.
\end{abstract}

Ziel des vorliegenden Beitrags ist es, dieses Phänomen näher zu beleuchten. Daher soll zunächst das Verhältnis von Fiktionalität und Faktualität in Das siebte Kreuz geklärt und dann die Symbolik des Romans erläutert werden. Im dritten Schritt ist die im Text zur Anschauung gelangende Historisierung zu analysieren.

Die Untersuchung wird einen im weitesten Sinne hermeneutischen Ansatz verfolgen, um so die vielfältigen zeitgeschichtlichen Bezüge von Das siebte Kreuz angemessen einzuordnen. Am Ende dieser Untersuchung lässt sich zeigen, dass die Historisierung in diesem Roman vor allem ein Mittel ist, um die Universalität bestimmter Themen sichtbar zu machen.

Schlüsselwörter: Anna Seghers, Historisierung, Nationalsozialismus, Fakten, Fiktionen, Symbolik.

1 Dieser Artikel ist Teil meiner Magisterarbeit Historisierung in der Exilliteratur. Fallbeispiele: Bertolt Brecht, Anna Seghers und Thomas Mann (2017).

2 Doktora Öğrencisi, Trakya Üniversitesi-Namık Kemal Üniversitesi, Alman Dili ve Edebiyatı Anabilim Dalı, burcuozturrk@gmail.com. 


\section{ANNA SEGHERS'IN YEDINCI HAÇ ROMANINDA TARIHSELLEŞTIRME ÜZERINE}

Öz: Anna Seghers'in Yedinci Haç adlı romanında Üçüncü Alman İmparatorluğu toplumu betimlenmektedir. Sürgün Edebiyatı için tartışmasız bir öneme sahip olan Anna Seghers, Yedinci Haç adlı romanında Almanya'daki nasyonalsosyalizmi anlatmak için gerçek ve kurguyu sembollerle birleştirmiş̧ir. Esas olarak Anna Seghers Yedinci Haç romanında insanlığın kültürel ve tarihi sürecini anlatmıştır. $\mathrm{Bu}$ sebeple metinde tarihselleştirme olarak adlandırılabilecek bir olgu ortaya çıkmıştır. Romanda tarih, içerisinde daima kazananların ve kaybedenlerin olduğu ya da başka bir ifadeyle kazananların herhangi bir zamanda, bir gün kaybeden olacağı görülmektedir. Simgesel sayılar ve Hristiyan inancına ait olan, dini bir sembol olan haçın simgesel olarak kullanılması gibi tarihselleştirme olgusunun farklı örnek kullanımları ve bu örnekler aracıllğıyla Anna Seghers'in betimlediği sorun evrensel bir boyut kazanır. Makalenin amacı tarihselleştirme olarak nitelendirilen bu olguyu daha ayrıntılı olarak açıklamaktır. Bu nedenle Anna Seghers'in Yedinci Haç eserindeki kurgu ve gerçeklik arasındaki ilişki öncelikle açıklığa kavuşturulacak ve daha sonra da romanın simgeselliği açıklanacaktır. Bundan sonra da, metinde yer alan tarihselleştirme irdelenecektir. Araştırma, Yedinci Haç eserinin çok yönlü tarihsel ilişkilerini uygun bir şekilde sınıflandırmak için geniş anlamda hermetik yaklaşım tekniği (yorumlama) ile yürütülecektir. Çalışmanın sonunda ise tarihselleştirme olgusunun bazı konuları görülebilir kılmak için kullanılan bir araç olduğu gösterilecektir.

Anahtar Sözcükler: Anna Seghers, Tarihselleştirme, Nasyonal Sosyalizm, Kurgu, Gerçek, Sembolik.

\section{Einführung}

Anna Seghers, die mit bürgerlichem Namen Nennty Reiling hieß, wurde 1900 in Mainz als die einzige Tochter von jüdischen Eltern geboren. 1933, nach der Machtübernahme Hitlers, floh sie mit ihrer Familie nach Frankreich. Während der Zeit im Exil schrieb Anna Seghers über viele Themen, wie Faschismus, Leben im Exil, Nationalsozialismus u.a. Eines ihrer berühmtesten Werke ist Das siebte Kreuz, das vielleicht den Höhepunkt in ihres Schaffens markiert.

Die ersten Seiten dieses Romans erschienen 1939 in der in Moskau herausgegebenen Exilschrift Internationale Literatur. 1942 erschien das Buch in englischer Sprache als The seventh cross in Boston mithilfe des mexikanischen Exilverlags El Libro Libre. Im selben Jahr, 1942, gab 
dieser Verlag das Buch in deutscher Sprache heraus. 1946 erschien das Buch zum ersten Mal in Berlin.

In Das siebte Kreuz hat die Geschichte deutliche Spuren hinterlassen. Der Roman Das siebte Kreuz von Anna Seghers fand in Deutschland zur Zeit des Nationalsozialismus statt. Es lässt sich feststellen, dass Anna Seghers in diesem Roman dem Leser ein realitätsnahes Bild von Deutschland um 1937 präsentiert. Aus dem Exil versuchte sie, soweit es ihr möglich war, die Situation in Deutschland darzustellen. Anhand der sieben Flüchtlinge, von denen nur einer, Georg Heisler, Erfolg hatte, zeigte sie einen Hoffnungsschimmer.

Das siebte Kreuz hat einen überaus präsenten zeitgeschichtlichen Hintergrund. Die gelebten Ereignisse in Deutschland zwischen 19331945 werden im Text sozusagen abgebildet. Das Hauptthema ist die Flucht von sieben Häftlingen: Georg Heisler, Beutler, Wallau, Aldinger, Pelzer, Füllgrabe und Belloni. In diesem Roman dauert die Erzählung ihrer Flucht sieben Tage und außerdem bekommt man einen Eindruck von der Atmosphäre der Konzentrationslager dieser Zeit und der Gesellschaft des ,Dritten Reiches ${ }^{\text {. }}$

Um die historische Realität der Zeit zu authentifizieren, nennt Anna Seghers den Namen Hitlers (1997, S. 170). Z.B. sagt ein Mann im Konzentrationslager:

Gleich im ersten Monat der Hitlerherrschaft [Herv. d. Verf.] hatte man Hunderte unserer Führer ermordet, in allen Teilen des Landes, jeden Monat wurden welche ermordet. Teils wurden sie öffentlich hingerichtet, teils in den Lagern zu Ende gequält.

Zudem wünschen sich die Menschen auf den Straßen keinen „Guten Tagee, sondern sie benutzen den Hitlergruß „Heil Hitler". Das ist auch der Teil des Alltags im ,Dritten Reich`. Die Vorschrift, immer mit „Heil Hitlere zu grüßen, bezeugt einen großen sozialen Druck. Der überall ertönende Hitlergruß gehörte zur Normalität. Das Volk hörte immer „heil Hitler hin, heil Hitler her" (Seghers, 1973, S. 329).

Die Familie Röder wird als unpolitische Arbeiterfamilie dargestellt. Sie denken, dass sie Glück haben.(Ohlsen, 2017, S.88):

Es ist jetzt wirklich nicht mehr so schwer' sagte die Liesel Röder, ,Kinder zu bekommen.' - ,Das war's ja nie.' - ,Ach, Georg ' rief Liesel, ,dir kommen deine Lebensgeister zurück.' - ,Nein, es ist wahr, wir waren fünf zu Haus, und ihr? - ,Der Fritz, der Ernst, ich und der Heini - vier.' - ,Nie hat ein Hahn nach uns gekräht.' sagte Liesel. ,Jetzt geschieht doch was.' Paul sagte mit lachenden Augen: ,Liesel hat einen staatlichen Glückwunsch von der Direktion bekommen.' - ,Habe ich bekommen, ja, 
ich! ${ }^{e}$ - ,Soll man dich vielleicht für die große Leistung beglückwünschen? - ,Spaß beiseit, Georg, all die Vergünstigungen und die Zulagen, sieben Pfennig pro Stunde, das spürst du. Die Befreiung von den Abzügen und ein solcher Stoß bester Windeln!' - ,Als ob die NSVolkswohlfahrt geahnt hätte ${ }^{e}$ sagte Paul, [...] dem Paul seine Äugelchen haben ganz schön gefunkelt, er war fiedel wie in seiner Bräutigamszeit, diesen August auf der Sommerreise- ' -,- , Wo ging's denn hin? - , nach Thüringen, [...] (Seghers, 1997, S. 246f)

Nach diesem Zitat der Familie Röder versteht man, dass das Volk vom NS-Staat begeistert war, der ihm spürbare Vorteile verschaffte: im Zitat spricht Paul von Vergünstigungen, Zulagen, Steuerleichterungen und nützlichen Geschenken (,,und ein solcher Stoß bester Windeln!ee). -,,Jetzt geschieht doch was ${ }^{\text {ee }}$ - Liesel spricht hier wahrscheinlich aus, was andere, die sich früher vom Staat verlassen fühlten, über die politischen Veränderungen gedacht haben mochten. Obwohl im Text viele über die gegenwärtigen Verhältnisse erfreut sind, gibt es doch auch einige Familien, auf die das nicht zutrifft. Dies kann anhand des Beispiels der Familie Fiedler erläutert werden. Anna Seghers (1997, S. 369) beschreibt die Einstellung dieser Familie so: „Im Dritten Reich hatten sie keine Kinder gewollt, weil sie dann später in braune Hemden gesteckt würden und zu Soldaten gedrillt." Man kann also sagen, dass Anna Seghers ein alltägliches Bild des nationalsozialistischen Deutschland zeichnet, indem sie nahe an der geschichtlichen Realität bleibt.

Wie ich im Titel der vorliegenden Arbeit andeute, geht es mir besonders darum, ein im Roman zur Anschauung gelangendes Phänomen zu erfassen, das ich Historisierung nennen möchte. Daher werde ich mich zunächst dem zuwenden, was entschieden $\mathrm{zu}$ den historisierenden Momenten in Das siebte Kreuz beiträgt: dem Spiel von Fakten und Fiktionen auf der einen und der Symbolik auf der anderen Seite. Da es sich bei dem Text nicht nur um ein literarisches, sondern in gewisser Weise auch zeitgeschichtliches Dokument handelt, wird die Untersuchung einen im weitesten Sinne hermeneutischen Ansatz verfolgen.

\section{Fakten und Fiktionen in Das siebte Kreuz}

Aufgrund der vielfältigen zeitgeschichtlichen Bezüge von Das siebte Kreuz stellt sich konsequenterweise die Frage, wie im Text das Verhältnis von Fakten und Fiktionen gestaltet wird. Bernhard Spies (1997, S. 38) zufolge beeindruckt dieser Roman den Leser durch ein ,vielfältiges Mosaik von Szenen aus dem Alltag."

Im Grunde beginnt bereits mit den Namen der KZ Osthofen/Westhofen ein fiktional-faktuales Spiel. Thilo Weckmüller spricht in diesem 
Zusammenhang von der ,literarischen Verfremdung” der historischen Vorlage:

Die Schriftstellerin Anna Seghers setzte den in Osthofen inhaftierten Menschen ein weltberühmtes literarisches Denkmal. Ihr Roman ,Das siebte Kreuz nimmt seinen Beginn in dem Lager, das Seghers der literarischen Verfremdung wegen,Westhofen ${ }^{\circ}$ nennt. Sie schildert die Flucht von sieben Häftlingen, die letzlich nur einem von ihnen gelingt. ${ }^{3}$

Das KZ Westhofen hat also - dies signalisiert bereits die an das historische Osthofen angelehnte Namensgebung - im Hinblick auf die Fiktionalität des Romans eine wichtige Funktion. Aus historischen Quellen geht hervor, dass die Häftlinge in Osthofen meistens vier bis sechs Wochen blieben. Aber der Protagonist dieses Romans, Georg Heisler, bleibt fast vier Jahre im KZ Osthofen! Normalerweise, d.h. in der Realität, wäre dies unmöglich gewesen.

Barbara Ritter ${ }^{4}$ erklärt, dass in den ersten Jahren vom Bestehen der Konzentrationslager der ehemaligen Papierfabrik als KZ genutzt wurden, damit die Gefangenen zu besiedeln. Die Lebensbedingungen waren menschenunwürdig:

Von 6 März 1933 bis Juli 1934 wurden die Gebäude der ehemaligen Papierfabrik als ein frühes Konzentrationslager (des damaligen Volksstaates Hessen) genutzt. Ohne richterliche Verfügung verhafteten Hilfspolizisten missliebige Personen und politische Gegner des NSRegimes, allen voran Mitglieder der KPD, der SPD und Gewerkschafter, aber auch Angehörige des Zentrums, Juden, Zeugen Jehovas und Sinti. Die Gefangenen waren in der einstöckigen Halle links des Tores untergebracht. Die hygienischen Verhältnisse und die Unterbringung in der zugigen und nasskalten Fabrikhalle waren äußerst primitiv. Zynisch wurde in der NS- Presse, die häufig darüber berichtete, das KZ Osthofen als Umerziehungslager für ,,verwilderte Marxisten“ bezeichnet, mit der Verköstigung „,besser als bei Muttern“. Zwar wurde im KZ Osthofen in den 14 Monaten seines Bestehens kein Häftling ermordet, aber die Gefangenen wurden menschenunwürdig misshandelt. Viele der Inhaftierten wurden nach der Schließung des Lagers erneut verfolgt, in andere Haftstätten und Lager verschleppt und später auch getötet.

In den Lagern wurden also keine Häftlinge ermordet, auch wenn die Haftbedingungen menschenunwürdig waren - so sah es in der Realität aus. In der fiktiven Welt des Romans hingegen will der

\footnotetext{
${ }^{3}$ Vgl. http://www.widerstand-portrait.de/portraits/kz-osthofen.html (Stand: 14.10.2017)

${ }^{4} \mathrm{Vgl}$. http://www.rhein-neckar-industriekultur.de/objekte/papier-und-möbelfabrik-heutegedenkstätte-kz-osthofen (Stand: 15.10.2017)
} 
Lagerkommandant Fahrenberg die geflüchteten Häftlinge, sobald sie gefasst sind, töten lassen.

Mit Fahrenberg hat es in diesem Zusammenhang eine besondere Bewandtnis. Im realen Leben führte nämlich ein gewisser Karl D’Angelo das Konzentrationslager in Osthofen. In Das siebte Kreuz wird er von Fahrenberg repräsentiert. So lässt sich natürlich auf Parallelen zwischen diesen beiden Lagerkommandanten schließen. Karl D'Angelo kann folgendermaßen charakterisiert werden:

Persönliche massive Beteiligungen an den Misshandlungen von Gefangenen werden ihm nicht nachgesagt. Er verhinderte aber auch nicht, dass im KZ Osthofen die Menschenwürde mit Füßen getreten wurde und Misshandlungen sowie Schikanen an der Tagesordnung waren. Als überzeugter Nationalsozialist und Antisemit versuchte Karl D'Angelo in vielen Gesprächen, politische Gegner zur NS-Ideologie zu bekehren. Mit jüdischen Häftlingen diskutierte er jedoch nicht und ließ deren besonders unwürdige Behandlung im Lager zu. (Tvrzníková, 2012, S. 35)

Fahrenberg hingegen wird gleich zu Beginn von Das siebte Kreuz wie folgt charakterisiert: „War der erste Kommandant ein Narr gewesen, mit furchtbaren, unvoraussehbaren Fällen von Grausamkeit, [...] Fahrenberg war imstande gewesen, uns plötzlich alle zusammenschlagen zu lassen- ${ }^{-e}$ 244 (Seghers, 1997, S.9)

Außerdem wird Fahrenberg beschrieben als „der alte Kämpfer, der Eroberer von Seelingenstadt, wo sein Vater noch heute ein Installationsgeschäft am Marktplatz hat ${ }^{\text {ec }}$ (Seghers, 1997, S. 9). Zum Vergleich: D'Angelo wurde 1890 in Osthofen geboren und 1918 hat er ähnlich wie Fahrenberg - die Druckerei seines Vaters übernommen. Er machte übrigens ebenfalls Karriere in der NSDAP und wurde Führer von der Schutzhaftabteilung im KZ Dachau. Genauso wie Fahrenberg wurde er auch abgesetzt. Wie man sieht, sieht Fahrenberg D'Angelo in vielen Punkten sehr ähnlich. Zugleich ist er aber mit Merkmalen ausgestattet, die ihn grausamer als sein historisches Vorbild erscheinen lassen. So kann man sagen, dass hier die Fakten gleichsam nur das Gerüst sind, auf dem Seghers die fiktionalen Inhalte von Das siebte Kreuz aufbaut.

Eine andere Parallele zwischen Fiktion und Realität ergibt sich aus der Flucht von einem der Häftlinge, nämlich Wallau, der zugleich ein Freund des Protagonisten Georg Heisler ist. Die Verhaftung Wallaus kommt im Text wie folgt zur Sprache:

Fischer rief: „Sie haben den Wallau. " Overkamp langte sich den Hörer, er kritzelte. „Ja, alle vier"e, sagte er. Dann sagte er: „Wohnung versiegeln." Dann: „Herbringen." [...] Wallaus Frau hat Sachen in seiner Laube bei Worms untergestellt, will nicht gewußt haben, wozu und was, unter 
Beobachtung wieder heimgelassen worden zwecks Beobachtung weiteren Verkehrs. Wallau um dreiunzwanzig Uhr zwanzig auf diesem Laubengrundstück verhaftet, verweigert bis jetzt jede Aussage. (Seghers, 1997, S.153)

Den historischen Bezugspunkt stellt hier die Verhaftung des KPDVorsitzenden Ernst Thälmann dar, der

zweifellos eine der großen Leitfiguren der deutschen Kommunisten im 20. Jahrhundert und das wichtigste politische Vorbild der Partei Ulbrichts [war]. [...] Der 1886 geborene Ernst avancierte in den 1920er Jahren zum Vorsitzenden der Kommunistischen Partei Deutschlands. Nach Hitlers Machtübernahme 1933 wurde er verhaftet und verbrachte 11/1/2 Jahre in Einzelhaft, bis ihn die SS 1944 ermordete. (Börrnert, 2004, S.11)

Wallau und Ernst Thälmann haben gleichen Lebenslauf. Deswegen kann man sagen, dass in Das siebte Kreuz Thälmann von Wallau repräsentiert wird.

Neben diesen Beispielen gibt es eine wichtige Ähnlichkeit zwischen dem Protagonisten des Romans, Georg Heisler, und Hans Beimler, dem Mitglied der Kommunistischen Partei Deutschlands. Im Nachfolgenden sollen die Besonderheiten beider Männer detailliert beschrieben werden.

Georg Heisler ist einer der sieben Häftlinge, der bei der Flucht Erfolg hat. Seine erfolgreiche Flucht lässt ihn zum Helden des Romans werden. Auf diese Weise wird er zur Hoffnung der anderen Gefangenen. Hans Beimler ist ein realhistorisches Vorbild für den Protagonisten Georg Heisler. Man weiß folgendes über Hans Beimler:

Hans Beimler, eigentlich Johann Evangelist Beimler, wurde am 2. Juli 1895 in München als Sohn der ledigen Köchin Rosina Beimler geboren. [...] Hans kam zunächst in das Polizegefängnis München, wo er brutal misshandelt wurde. Am 25. April 1933 wurde er in das neu erbaute KZ Dachau überstellt. Dort gelang es ihm in der Nacht vom 8. auf den 9. Mai 1933, zu fliehen. Zunächst fand er bei Freunden Unterschlupf, bevor er über München nach Prag und schließlich in die Sowjetunion fliehen konnte. [...] 1934 entzogen die Nazis Hans Beimler die deutsche Staatsbürgerschaft. [...] Am 01. Dezember 1936 traf ihn in Madrid eine Kugel, die offenbar ein faschistischer Scharfschütze abgefeuert hatte. Mehr als zwei Millionen Menschen nahmen in Madrid Abschied von Hans Beimler. Beigesetzt wurde er auf dem Bergfriedhof Montjuïc in Berlin. $^{5}$

Neben dieser Flucht von Hans Beimler ist die Flucht von Max Tschornicki vergleichbar mit der Heislers im Roman. Um die Wichtigkeit

\footnotetext{
${ }^{5}$ Vgl. http://www.hans-beimler-zentrum.de/hans-beimler/ (Stand: 16.10.2017)
} 
dieser Person für die Zeichnung des Protagonisten zu illustrieren, lässt sich das Leben von Tschornicki als Beispiel anführen:

Der in Rüsselsheim geborene Max Tschornicki wuchs in einem orthodox jüdischen Elternhaus in Mainz auf. [...] Er war Mitglied der Jungsozialisten, der SPD und der sozialdemokratisch orientierten Schutzorganisation „Reichsbanner Schwarz- Rot- Gold“e, deren Mitglieder er später als Jurist vor Gericht verteidigte. 1933 wurde Max Tschornicki mehrfach verhaftet und kurzzeitig inhaftiert. Am 24. Mai 1933 wurde er auf Grundlage der ,,Verordnung zum Schutz von Volk und Staat" in das KZ Osthofen eingeliefert, aus dem ihm am 3. Juli 1933 die Flucht gelang. Max Tschornicki floh zunächst in das Saargebiet und nach dessen Angliederung an das Deutsche Reich nach Frankreich. In Toulouse erfuhr er von der Verhaftung seiner Verlobten und deren Mutter, für deren Freilassung er sich einsetzte. Max Tschornicki wurde nach der Besetzung Südfrankreichs in Lyon, wo es zu dieser Zeit eine starke politisch- jüdische Widerstandsbewegung gab, von der Gestapo verhaftet. Max Tschornicki überlebte eine Odysee durch mehrere Konzentrationslager bevor er am 20. April 1945 in Allach, einem Außenlager von Dachau, wenige Tage vor der Befreiung des Lagers durch Truppen der US- Armee starb. ${ }^{6}$

Zusammenfassend kann festgehalten werden, dass Fakten und Fiktionen im Siebten Kreuz aufs Engste miteinander verschränkt sind. Wie gesehen, gibt es auffallende Ähnlichkeiten nicht nur zwischen bestimmten Namen (KZ Osthofen/KZ Westhofen), sondern auch Charakteren des Figurenpersonals und realhistorischen Personen. Da die Protagonisten des Romans im Grunde stellvertretend für alle Gefangenen im ,Dritten Reich ${ }^{\text {}}$ stehen, kann man sagen, dass sie mit ihren Lebensgeschichten nahezu symbolische Bedeutung haben - womit das Thema des nächsten Abschnitts erreicht wäre.

\section{Symbolik in Das Siebte Kreuz: ,Sieben ${ }^{e}$ und ,Kreuz}

Die Erzähltechnik des Romans Das siebte Kreuz hängt stark mit der Verwendung von Symbolen zusammen, mit denen Seghers das NaziRegime darstellt. Nach Erhard Schütz und Jochen Vogt hat „Das siebte Kreuz [...] eine novellistische Grundstruktur. Das quer durch alle Klassen und Schichten gelegte Gesellschaftspanorama zu gestalten, widersprach ihrer erzählerischen Methode der Unmittelbarkeit mit eingelagerter Geschichtssymbolik." (Schütz und Vogt, 1980, S. 41)

Als ein Hauptmotiv wählte Anna Seghers in ihrem Roman Das siebte Kreuz die Zahl ,Sieben'. In dem Roman ist es in vielfältiger Form

\footnotetext{
Vgl. http://www.projektosthofen-gedenkstaette.de/index.php?page=42 (Stand: 16.10.2017)
} 
präsent: Der Roman hat sieben Kapitel. Sieben Häftlinge fliehen aus dem Konzentrationslager, weshalb der Lagerkommandant Fahrenberg sieben Kreuze aus den Platenen bilden lässt. Zusätzlich hat er sieben Tage, um die Häftlinge zu finden. Dass es Georg Heisler schafft, sich am siebten Tag zu retten, ist ebenfalls interessant. Man kann also davon ausgehen, dass die Zahl ,Sieben` ein Leitmotiv des Textes ist. Laut Petra Neumayer (2011, S. 32) ergeben

[d]ie 3 und die 4 [...] die 7: Der Geist hat sich mit der Materie verbunden. Geistliches und Weltliches bilden eine Synthese. In sechs Tagen erschuf Gott die Welt, am siebten Tag ruhte er. In der Heiligen Geometrie offenbart sich die erste Blume des Lebens, die Saat ist aufgegangen: Die 7 ist eine weitere Entfaltung der Schöpfung, entsprungen aus der 1. Zugeordnetes Symbol ist die erste Blume des Lebens, die die Liebe alles Seins repräsentiert.

Hier entsteht die Frage: Welche Rolle diese Zahl im literarischem Kontext spielt. Am siebten Tag schafft es Georg Heisler, sich zu retten, wodurch sein neues Leben beginnt. Vor diesem Hintergrund ist anzunehmen, dass der Anfang des neuen Lebens mit der Zahl ,Sieben symbolisiert wird. Tatsächlich erfüllt Georg Heislers Flucht die anderen Gefangenen mit Hoffnung. „Wir fühlten alle”, so der Erzähler, der bekanntlich einer der Gefangenen ist, ,,wie tief und furchtbar die äußeren Mächte in den Menschen hineingreifen können, bis in sein Innerstes, aber wir fühlten auch, daß es im Innersten etwas gab, was unangreifbar war und unverletzbar." (Seghers, 1997, S. 425) Die Zahl ,Sieben“ symbolisiert also die Hoffnung auf Neuerung.-Ferner meint der Erzähler:

Unser Gefühl, das wir nicht verbergen konnten, reizte die Peiniger noch mehr. So stark empfanden die meisten von uns diese Flüchtlinge als einen Teil von uns selbst, daß es uns war, als seien sie von uns ausgeschickt. Obgleich wir nichts von dem Plan gewußt hatten, kam es uns vor, etwas Seltenes sei uns gelungen. Vielen von uns war der Feind allmächtig vorgekommen. Während die Starken sich ruhig einmal irren können, ohne etwas zu verlieren, weil selbst die mächtigsten Menschen noch Menschen sind - ja sogar ihre Irrtümer machen sie nur noch menschlicher -, darf sich, wer sich als Allmacht aufspielt, niemals irren, weil es entweder Allmacht ist oder gar nichts. Wenn ein noch so winziger Streich gelang gegen die Allmacht des Feindes, dann war schon alles gelungen. (S. 169f)

In diesem Zusammenhang kann man sagen, dass Georg Heisler für seine Freunde wie eine Kultfigur ist, die ihnen Hoffnung gibt. Seine erfolgreiche Flucht wird so zu einem Symbol des Widerstandes. Sie lässt sich darüber hinaus auch als Zukunftshoffnung begreifen. Deshalb hat sie nicht nur für Georg Heisler, sondern auch für alle anderen Gefangenen 
eine existenzielle Bedeutung. Sie bedeutet nämlich so etwas wie „,bewahrte Humanität" (Huml und Rappenecker, 2003, S. 252).

Neben der Zahl ,Sieben ' bildet im Roman natürlich auch das titelgebende „Kreuz ein wichtiges Motiv. Normalerweise ist dieses Zeichen den Christen bekannt, steht es doch im Zentrum der christlichen Symbolik: „Das Kreuz ist das Zeichen der Befestigung aller Menschen ,auf Christus', der Besiegelung, mit dem heiligen Geist der Verheissung." (Bunsen, 1876, S. 235) Tatsächlich hat das Kreuz etwas Grausames an sich. Durch den Tod Christi erlangte es fragwürdige Berühmtheit. In der einschlägigen Stelle in der Bibel heißt es:

Was soll ich mit Jesus tun, den man Christus nennt? Sie sprachen alle zu ihm: Kreuzige ihn! Da sagte der Statthalter: Was hat er denn Böses getan? Sie aber schrien noch viel mehr und sprachen: Kreuzige ihn! [...] „,Dies ist Jesus, der König der Juden." Dann wurden mit ihm zwei Räuber gekreuzigt, einer zur Rechten, der andere zur Linken. (Schlachter, 2000, S. 1029 f.)

Da Jesus Christus indes der Bibel zufolge wiederauferstanden ist, könnte das Kreuz auch Hoffnung auf Neuerung, ein neues Leben u.ä. symbolisieren.

Einen zentralen Punkt in Seghers Roman stellt ,das leere Kreuz dar: es ist eben jenes siebte für Georg Heisler bestimmte Kreuz, das leer bleibt. Man kann davon ausgehen, dass Seghers so auch auf ein mögliches Ende des Nationalsozialismus hindeuten wollte, quasi als Hoffnung für den Leser bzw. die „toten und lebenden Antifaschisten Deutschlands”, wie Seghers in der Widmung schreibt. Entsprechend meint Birgit Ohlsen (2017, S. 62): „Das siebte Kreuz bleibt leer und wird somit zum Symbol der Überwindbarkeit des Faschismus." Ähnlich symbolisiert für Heck und Wöbcke (2016, S. 125) das leere Kreuz den unausweichlichen Untergang des Nationalsozialismus. Ferner kann mas das leere Kreuz wie folgt interpretieren:

Abgesehen von der Symbolik des leer bleibenden siebten Kreuzes, das bedeutet, dass das Nazi-Regime das Volk nicht besiegen kann, sondern sich selbst zu Grunde richten wird, ist die entscheidende Botschaft der Geschichte die Bedeutung der Solidarität." (Schmidt und Tallafus, 2007, S. 25)

In diesem Zusammenhang überrascht den Leser ein anderes Symbol des Romans, nämlich das Konzentrationslager, das in gewisser Weise das ,Dritte Reich ${ }^{`}$ symbolisiert. Denn erst mit dem Hitler-Regime tauchten die Konzentrationslager in Deutschland auf, hängen also unauflöslich damit zusammen. Meiner Meinung nach symbolisiert das Konzentrationslager die Grausamkeit des Hitler-Regimes. Zudem 
symbolisiert es die Hoffnungslosigkeit der Menschen in den Jahren zwischen 1933-1945.

\section{Die Historisierung in Das siebte Kreuz}

Der Nationalsozialismus stellt in der deutschen Geschichte eine deutliche Zäsur dar. Obwohl er nur zwölf Jahre andauerte, hat er ohne Zweifel Spuren in der deutschen Kultur hinterlassen. So gesehen handelt Das siebte Kreuz in gewisser Weise auch vom kulturellen und historischen Prozess der Menschheit.

Im Vordergrund des Textes steht die erfolgreiche Flucht Georg Heislers bzw. die Revolte gegen ein unmenschliches Regime, das vom KZ Westhofen repräsentiert wird. Zwar spielt der Kommunismus eine tragende Rolle im Roman, doch ist es nicht von der Hand zu weisen, dass Seghers hier den Unterschied zwischen den Menschen als etwas Historisches bzw. Vergängliches darstellt. In Das siebte Kreuz erscheint Geschichte als ein endloser Prozess von Gewalt, in dem es immer Sieger und Besiegte gibt. Oder anders ausgedrückt: der Roman akzentuiert, dass in der Geschichte Sieger immer irgendwann zu Besiegten werden. Genau hierin liegt die Bedeutung des im Text entstehenden Phänomens, das hier eingangs als Historisierung bezeichnet wurde. Historisierung bedeutet zunächst einmal „Aufarbeitung der Vergangenheit" (Vilar, 2004, S. 9), bei der man davon ausgehen kann, dass sie „schöpferisch mit den Beziehungen zwischen Vergangenheit und Gegenwart arbeitet ${ }^{\text {ee }}$ (Hoogeveen und Labroisse, 1981, S. 241). Bettina Bannasch und Gerhild Rochus zufolge (2013, S. 198) betrifft Historisierung

den Zusammenhang mit der geschichtlichen und kulturellen Überlieferung. Sie bezieht sich gleichermaßen auf die Gegenwart. Die Erfahrungen der gegenwärtigen Leser und Interpreten, der zeitgeschichtliche Kontext aktueller Exile und die Antworten, die die Gegenwart - die Gesellschaft, die Politik, die Wissenschaft und die Literatur - darauf finden, bilden den Erfahrungshorizont, von dem aus der Blick der Leser und Forscher sich zurück auf das historische Exil richtet.

Demgemäß eignet sich die Historisierung in Das siebte Kreuz nicht nur zur Erfassung der Vergangenheit, sondern auch und vor allem zur Vorführung der Gegenwart.

Auf dieser Grundlage rücken im Roman die vielfältigen Beziehungen zwischen der Vergangenheit und Gegenwart in den Fokus. Durch (symbolische) Anspielungen auf die Vergangenheit (wie etwa durch das ,Kreuz') nimmt der Leser eine generalisierende Perspektive auf die beschriebene NS-Zeit ein. Mithin gewinnen die zeitgenössischen Themen, die im Text zur Darstellung kommen, eine universale 
Dimension, die durch die Kreuz- und Zahlensymbolik zusätzlich unterstrichen wird.

Aus dieser historisierenden Perspektive erscheinen im Roman die durch den Nationalsozialismus verursachten Misstände weniger als politisches denn vielmehr menschliche Probleme. Deshalb ist der Nationalsozialismus im Roman auch nicht so sehr als eine bestimmte politische Ideologie zu verstehen, denn durch die Historisierung erscheint er als Repräsentant der Macht an sich. So kann der Leser von Das siebte Kreuz in gewisser Weise Zeuge werden, wie rücksichtlos die ,Macht ${ }^{\mathrm{e}}$ gegen die Menschen - oder die Menschheit - vorgeht, um sich selbst zu erhalten. Er kann aber auch sehen, dass es dem Menschen möglich ist, sich aufzubäumen und Widerstand zu leisten.

Im Übrigen ist die Historisierung etwas, das Seghers' Roman stark mit dem literaturgeschichtlichen Kontext der Exilliteratur verbindet, in dem er entstand. Laut Wiebke von Bernstorff (2006, S. 57) spielen literarische Texte, die im Exil geschrieben wurden,

fast ausnahmlos nicht in Deutschland. Es lassen sich in den Texten grob zwei Linien unterscheiden, die von einer beträchtlichen Spannung gekennzeichnet sind. Auf der einen Seite stehen die Erzählungen, die entweder von einer mythischen Vorzeit oder von fremden Ländern und historisch entlegenen Zeiten sprechen. Auf der anderen Seite finden sich didaktische Erzählungen in politisch konformer Manier.

Zwar beschreiben die Exil-Autoren zumeist die eigene Gegenwart, jedoch benutzen sie dabei sehr häufig die Vergangenheit oder den Mythos als literarische Hintergrundfolie. Man denke nur an Thomas Manns Romantetralogie Joseph und seine Brüder, worin der Versuch einer „Humanisierung des Mythischen“ bzw. die Fokussierung auf das „EwigMenschliche" ebenso wie die spezifische Form der Historisierung, durch die der dem Joseph eigene Reflexionsmodus entsteht, eine tragende Rolle spielen (Bazarkaya und Öztürk, 2017, S. 66 ff.). Es ist also festzuhalten, dass in Texten der Exilliteratur die soziale und politische Situation Deutschlands - nicht zuletzt mittels der Historisierung - in hohem Maße literarisiert bzw. fiktionalisiert wird.

Ein Beispiel, in dem die Historisierung in Das siebte Kreuz besonders eindrücklich zur Anwendung kommt, ist die Stelle, wo Franz Marnet, ein Freund Georg Heislers, mit dem Rad ,von dem Gehöft seiner Verwandten, das zu der Gemeinde Schmiedtheim im vorderen Taunus gehörte, ein Paar Minuten früher als gewöhnlich auf seinem Fahrrad ab[fuhr]” (Seghers, 1997, S. 11). Nach Rüdiger Bernhardt ist „Der Vorgang sowohl real als auch symbolisch zu sehen (Bernhardt, 2009, S. 
19). Denn man kann diese Fahrt aus der hoch liegenden Idylle in die Tiefe quasi als Fahrt in die Hölle des nationalsozialistischen Alltags deuten. Wichtig ist im Zusammenhang der Historisierung vor allem die Rede vom Heiligen Römischen Reich Deutscher Nation. An der entsprechenden Stelle im Roman heißt es nämlich:

Bei der Mündung liegt Mainz. Das stellte dem Heiligen Römischen Reich die Erkanzler. Und das flache Land zwischen Mainz und Worms, das ganze Ufer war bedeckt von den Zeltlagern der Kaiserwahlen. [...] Zwanzig Jahre später stand auf der Mainzer Schiffsbrücke ein alter Soldat Posten. Wie sie an ihm vorüberzogen, die Letzten der Großen Armee, zerlumpt und düster, da fiel ihm ein, wie er hier Posten gestanden hatte, als sie eingezogen waren mit den Trikoloren und mit den Menschenrechten, und er weinte laut auf. Auch dieser Posten wurde zurückgezogen. Es wurde stiller, selbst hierzuland. Auch hierher kamen die Jahre 33 und 48, dünn und bitter, zwei Fädchen geronnenes Blut. Dann kam wieder ein Reich, das man heute das Zweite nennt. (Seghers, 1997, S.14f)

Rüdiger Bernhardt interpretiert diese Fahrt von Franz Marnet wie folgt:

Während der Fahrt wird die Geschichte der Landschaft von der mythischen Frühzeit über die römische Besetzung, die Christianisierung, das Heilige Römische Reich deutscher Nation und die Französische Revolution 1789 bis zum Zweiten Kaiserreich von 1871 und dem Beginn des Nationalsozialismus erinnert. (Bernhardt, 2009, S. 20)

Dadurch entsteht eine Brücke zwischen der Vergangenheit und Gegenwart. Denn Seghers stellt eine Kontinuität zwischen der NS-Zeit und früheren historischen Epochen her. Die ,Macht ${ }^{\circ}$, so könnte die Deutung lauten, war immer schon grausam und unmenschlich; solange es sie gibt, wird das - im Rückblick womöglich sinnlos erscheinende Blutvergießen fortdauern. Indem Seghers das NS-Regime aber quasi im Strom der Geschichte verortet, suggeriert sie, dass seine Macht beschränkt ist und irgendwann ganz verschwinden wird, so wie die Macht aller anderen Herrschaftssysteme, die längst untergegangen sind. Damit relativiert sie den Nationalsozialismus ebenso wie sie die Botschaft ihres Antifaschistischen Romans universalisiert.

\section{Fazit}

In dieser Arbeit wurde das Werk Das siebte Kreuz von Anna Seghers analysiert. Die Ausgangsfragen dieser Arbeit lauteten: „Was ist das Phänomen Historisierung? Welche Rolle spielt dabei der zeitgeschichtliche Hintergrund, um die Werke richtiger zu interpretieren?e Was Anna Seghers in ihrem Werk behandelt, kann folgendermaßen zusammengefasst werden: 
Der vorangegangenen Untersuchung liegt die Auffassung zugrunde, dass Anna Seghers mit Das siebte Kreuz ein Hitler-Deutschland darstellt, in dem Fiktionalität und Faktualität aufs Engste miteinander verschränkt sind. Seghers' Roman handelt nicht allein vom kommunistischen Widerstand gegen den Nationalsozialismus. Deshalb kommt dem Symbol des Kreuzes und der symbolischen Zahl ,Sieben eine so wichtige Bedeutung zu. Mit der Verwendung von Symbolen stellt Seghers das Nazi-Regime dar. Sie wählte die Zahl ,Sieben ${ }^{`}$ als ein Hauptmotiv. Der Anfang des neuen Lebens und die Hoffnung auf Neuerung werden mit dieser Zahl symbolisiert. Mit dem leeren Kreuz symbolisiert sie den Untergang des Nationalsozialismus. Dank dieser Techniken wird das dargestellte Thema in gewisser Weise universalisiert.

Seghers benutzt in ihrem Roman das Phänomen Historisierung und setzt sich so mit zeitgenössischen politischen Ereignissen auseinander. Durch die dabei zur Anwendung kommende Historisierung erscheinen die im Roman dargestellten Themen weniger als politische denn vielmehr als menschliche Probleme. Man kann also sagen, dass Das siebte Kreuz zwar ohne Zweifel „ein Roman des Widerstands" ist (Žmegač, 1994, S. 632), darüber hinaus aber auch eine universale Botschaft enthält, nämlich die, dass der Mensch sich unmenschlichen Regimen - ganz gleich, welcher politischen Richtung - widersetzen und den Kampf gegen sie nie aufgeben soll.

Zusammenfassend kann festgehalten werden, dass die vorangegangene Untersuchung ein ziemlich überraschendes Ergebnis erzielt hat. Es wurde nämlich gezeigt, dass die Historisierung in Seghers' Roman Das siebte Kreuz weniger bestimmte Ereignisse in der Vergangenheit fokussiert, als vielmehr dazu dient, aktuelles Zeitgeschehen zu universalisieren. In diesem Werk kann der Leser sehen, dass es dem Menschen möglich ist, sich aufzubäumen und Widerstand zu leisten.

\section{Literaturverzeichnis}

Bannasch, B. \& Rochus, G. (2013). Handbuch der deutschsprachigen Exilliteratur. Berlin: De Gruyter.

Bazarkaya, O. K. \& Öztürk, Burcu (2017): Auflösung des Medialen: Thomas Manns Joseph und seine Brüder und Richard Wagners Ring des Nibelungen. Tekirdağ: Humanitas, 5(10). S. 61-76.

Bernhardt, R. (2009). Erläuterungen zu Anna Seghers Das siebte Kreuz. Hollfeld: C. Bange Verlag.

Bernstorff, W. (2006). Fluchtorte Die mexikanischen und karibischen Erzählungen von Anna Seghers. Göttingen: Wallstein Verlag. 
Börrnert, R. (2004). Wie Ernst Thälmann treu und kühn! Das ThälmannBild der SED im Erziehungsalltag der DDR. Germany: Klinkhardt.

Bunsen, E. (1876). Das Symbol des Kreuzes bei allen Nationen und die Entstehung des Kreuz-Symbols der Christlichen Kirche. Berlin: Verlag von Mitscher\&Röstell.

Heck, G. \& Wöbcke, M. (2016). Dumont Reis Handbuch Reiseführer Mexiko: mit Extra-Reisekarte. Auflage 3. Köln.

Hoogeveen, J. \& Labroisse, G. (1981). DDR Roman und Literaturgesellschaft. Amsterdamer Beiträge zur neueren Germanistik Band 11/12-1981. Amsterdam: Rodopi.

Huml, A. \& Rappenecker, M. (2003). Jüdische Intellektuelle im 20.Jahrhundert Literatur- und kulturgeschichtliche Studien. Würzburg: Königshausen\&Neumann.

Neumayer, P. (2011). Heilen mit Zahlen von der Zahlenmystik bis zum spirituellen Codesystem- mit großem Praxisteil. Staffelsee: Mankau Verlag.

Ohlsen, B. (2017). „Heimat" im Exilwerk von Anna Seghers. Berlin: Frank\&Timme GmbH.

Schlachter, F. E. (2000). Die Bibel. Bielefeld: Christliche LiteraturVerbreitung.

Schmidt, H. J. \& Tallafuss P. (2007). Totalitarismus und Literatur: Deutsche Literatur im 20. Jahrhundert-Literarische Öffentlichkeit im Spannungsfeld totalitärer Meinungsbildung. Göttingen: Vandenhoeck\&Ruprecht.

Schütz, E. \& Vogt, J. (1980). Einführung in die deutsche Literatur des 20.Jahrhunderts. Band 3: Bundesrepublik und DDR. Opladen: Westdeutscher Verlag.

Seghers, A. (1973). Das siebte Kreuz. Hamburg: LuchterhandLiteraturverlag.

Seghers, A. (1997). Das siebte Kreuz. Ein Roman aus Hitlerdeutschland. Berlin: Taschenbuch Verlag.

Spies, B. (1997). Anna Seghers: Das siebte Kreuz: Grundlagen und Gedanken. Frankfurt am Main: Diesterweg.

Tvrzníková, J. (2012). Bernhard Schlinks „Der Vorleser" und Anna Seghers „Das siebte Kreuz" im Vergleich. Philosophische Fakultät der Masaryk Universität in Brünn. Magisterarbeit. Brünn. 
Vilar, L. (2004). Die Kritik des realen DDR- Sozialismus im Werk Anna Seghers: „Die Entscheidunge und „Das Vertrauen“. Würzburg: Königshausen\& Neumann.

Žmegač, V. (1994). Geschichte der deutschen Literatur vom 18. Jahrhundert bis zur Gegenwart. Band III/2. Weinheim: Beltz Athenäum Verlag.

\section{Internetverzeichnis}

Ritter, B. „Papier- und Möbelfabrik- heute Gedenkstätte KZ in Osthofen ${ }^{\text {ee }}$ http://www.rhein-neckar-industriekultur.de/objekte/papier-undmöbelfabrik-heute-gedenkstätte-kz-osthofen (Stand: 15.10.2017)

Weckmüller, T. „Trotz alledem! Ein Porträt des Widerstandes im RheinMain-Gebiet. Linoldrucke aus der Werkstaat uah! ${ }^{\text {ee }}$ http://www.widerstand-portrait.de/portraits/kz-osthofen.html (Stand: 14.10.2017)

http://www.hans-beimler-zentrum.de/hans-beimler/ (Stand: 16.10.2017) http://www.projektosthofen-gedenkstaette.de/index.php?page=42 (Stand: 16.10.20)

ON HISTORICIZATION IN ANNA SEGHERS' DAS SIEBTE KREUZ

Abstract: Anna Seghers' novel The Seventh Cross (1942) depicts the society of the Third German Empire. Anna Seghers, who has undeniable importance for the Exile literature, combines real and fictional symbols to describe national socialism in Germany in The Seventh Cross. In fact, in her novel The Seventh Cross Anna Seghers describes the cultural and historical process of humanity. In this way, something comes in the text, that can be referred to as a Historicization. In the novel, history seems like a power process, and there are the winners and losers in this process. Or in another words, it can be said that the winner will lose one day. Thanks to the different examples of the phenomenon of historicization, such as the use of symbolic numbers and symbolic use of the cross, the problem that Anna Seghers describes is of a universal dimension.

The aim of this work is to explain this phenomenon in detail. Therefore the relationship between fictionality and factuality in The Seventh Cross should be first clarified and then the symbolism of the novel is explained. The third step is to analyze the historicization in the text. The work will pursue a hermeneutical approach in the broadest sense, in order to classify the manifold historical references of The Seventh Cross 
appropriately. At the end of the work it will be shown that the use of historicization method makes certain themes noticeable.

Keywords: Anna Seghers, Historicization, National Socialism, Reality, Fiction, Symbolic. 